\title{
The Epithelial Basement Membrane Zone of the Limbus
}

\author{
ILENE K. GIPSON
}

Boston

\begin{abstract}
Summary
The basement membrane zone of the limbal epithelium adjacent to the cornea was examined by ultrastructural and immunohistochemical techniques to determine whether differences exist between this region and central cornea. In human limbus, the percentage of basal cell membrane occupied by hemidesmosomes was significantly less $(14.9 \pm 3.5)$ than that in central cornea $27.9 \pm 9.2)$, whereas the area of basement membrane/100 $\mu \mathrm{m}$ of cell membrane did not differ significantly. In rabbits, both percentage of membrane occupied by hemidesmosomes and area of basement membrane were less in the limbal region. Comparison of laminin and type VII collagen (anchoring fibril collagen) localisation in limbus and in central cornea demonstrated that both matrix proteins had a more convoluted pattern of localisation in the limbus. In addition, short segments of basement membrane with associated anchoring fibrils were present in the zone between the basal cells' basement membrane and blood vessels. These areas of duplicated basement membrane with anchoring fibrils were separated from the epithelium by layers of extracellular matrix that included collagen fibrils. Scanning electron microscopy of the surface topography of human limbal and central corneal basement membrane, prepared by removal of the epithelium with EDTA, demonstrated that in the limbal zone between the Palisades of Vogt and cornea, a very rough undulating surface was present with papillae or 'pegs' of stroma extending upward, and that central cornea lacked such papillae. Rabbit limbal basement membrane surface showed no such papillae, only occasional indentations into the stroma. Compared with central cornea, the differences in adhesion structure pattern in limbus may indicate that this surface has less abrasion pressure from the lids or that the convoluted interdigitation of cytoplasmic processes with the stroma compensates for the smaller number of hemidesmosomes.
\end{abstract}

Recent attention has focused on the limbal epithelium as a potential stem cell reservoir for the corneal epithelium. ${ }^{1,2}$ Unique properties have been ascribed to the limbal epithelium in that carbonic anhydrase and $\mathrm{Na} / \mathrm{K}$-ATPase are present within cells of this zone, ${ }^{3}$ and recently a monoclonal antibody to a $50 \mathrm{KD}$ protein was described that localises to the basal cells of the limbal epithelium. ${ }^{4}$ Tissues with unique cellular properties may synthesise different substrates to which the cells adhere. Having studied the adhesion structures of the corneal

Correspondence to: Ilene K. Gipson, PhD, Eye Research Institute, 20 Staniford Street, Boston, MA 02114, USA.

From the Eye Research Institute of Retina Foundation, and Department of Ophthalmology, Harvard Medical School, Boston, Massachusetts, USA.

This study was supported in part by grant EY03306 from the National Institutes of Health. 
epithelium in the differentiated,$^{5}$ developing ${ }^{6}$ and healing ${ }^{7}$ states, we have now examined these structures in the limbus.

The four adhesion structures of all stratified squamous epithelium, including the limbal epithelium, are hemidesmosomes, basement membrane, anchoring fibril network, and adhesion plaques. Hemidesmosomes are the cell-substrate adhesion junctions; they are linked through the basement membrane to the anchoring fibril network..$^{5,8}$ Anchoring fibrils terminate distal from the basement membrane in adhesion plaques. ${ }^{5,8}$ The only known component of the hemidesmosome is the bullous pemphigoid antigen (for review, see ref. 6). The corneal basement membrane contains laminin and the globular domain of type VII collagen $;{ }^{7}$ the presence of type IV collagen is still controversial. ${ }^{9}$ Anchoring fibrils insert into the basement membrane at sites opposite the sites at which hemidesmosomes are present along the basal cell membrane. ${ }^{6}{ }^{10}$ These uniquely crossbanded fibrils are composed of type VII collagen, a heterodimer of very high molecularweight in which each molecule has a large globular and a long helical domain. ${ }^{11.12}$ The globular domains associate and are present in the lamina densa and in anchoring plaques. Anchoring plaques are small patches of basement membrane-like material located in the stroma below the basement membrane in the zone of the anchoring fibril network. ${ }^{8}$ Helical domains of the molecules also associate and form the crossbanded pattern characteristic of anchoring fibrils.

We have examined by ultrastructural and immunohistochemical techniques the adhesion structures of human and rabbit limbal epithelium and compared the data with those previously obtained for the cornea. ${ }^{5}$ Specifically, we determined the ultrastructural arrangement of the hemidesmosomes and anchoring fibrils, the percentage of basal cell membrane occupied by hemidesmosomes, the area of basement membrane $/ 100 \mu \mathrm{m}$ of basal cell membrane, and the pattern of immunohistochemical binding of antibodies to laminin and type VII collagen. In addition, we studied by scanning electron microscopy the surface topography of human and rabbit limbal and corneal basement membrane that had been prepared by removal of epithelium by EDTA.

\section{Methods}

Ten human donor corneal rims were obtained from local surgeons. Age range of the donors was 17 to 77 years. The rims were cut in half, and one half was fixed in either half-strength Karnovsky's fixative or osmium collidine fixative and embedded by standard procedures for electron microscopy. ${ }^{5}$ The other half of the rim was frozen for cryosectioning and immunolabelling. Tissues from adult New Zealand white rabbits were processed in a similar manner.

Morphometric analysis of hemidesmosomes and basal lamina was done on electron micrographs from both species as previously described. ${ }^{5}$ Briefly, 10-12 micrographs of the basal cell basement membrane zone from each limbal sample were taken at a standard magnification at the region from the end of Bowman's layer and over the first blood vessels in from the cornea. For rabbit tissue (without a Bowman's layer), micrographs were taken at the region over the first blood vessels in from the cornea. Percentage of basal cell membrane occupied by hemidesmosomes and area of basement membrane $/ 100 \mu$ m of cell membrane were measured on electron micrographs using a Zeiss Videoplan digitiser. Two independent measurements were made and the data compared with those from normal central cornea. ${ }^{5}$ The Student's ' $t$ ' test was used for statistical comparison.

Procedures for immunohistochemical localisation on tissues of both species have been described elsewhere. ${ }^{5-7}$ Antibodies used were a polyclonal laminin antibody raised in sheep 5 and a monoclonal antibody to the globular domain of type VII collagen, generously supplied by Dr. Robert Burgeson of the Shriners Crippled Children's Hospital, Portland, Oregon. Secondary antibodies for the indirect procedure for localisation of laminin were rabbit and goat anti-sheep IgG in human and rabbit tissue, respectively, and goat antimouse IgG for localisation of type VII collagen.

To study the surface topography of the basement membrane of limbus and cornea, human donor rims or central buttons and rabbit cor- 
neas or cornea-limbal regions were incubated at $37^{\circ} \mathrm{C}$ for $1.5-2 \mathrm{hr}$ in $0.025 \mathrm{M}$ EDTA (tetrasodium salt) in Hanks balanced salt solution without $\mathrm{CA}^{2+}$ or $\mathrm{Mg}^{2+}$. The $\mathrm{pH}$ of the solution was brought to 7.4 with $2 \mathrm{M}$ monobasic sodium phosphate. After incubation, the epithelium was pulled off human donor rims or rabbit cornea-limbus by fine jewellers' forceps, starting at the cut edge of cornea inside the limbus and pulling toward the limbus. The epithelium was pulled off central cornea from the cut edge of the tissue. Tissues were fixed in half-strength Karnovsky's fixative, dehydrated in ethanol, critical point dried, and sputter-coated with gold. This technique is a modification of one described for epidermis. ${ }^{15}$

\section{Results}

The unique feature of the human cornea limbal zone, as compared with central cornea, is the interdigitation of basal cell processes between 'pegs' of stromal extracellular matrix. ${ }^{12}$ This is obvious by transmission electron microscopy ( Fig. 1), but the shape of the 'pegs' or papillae is most evident in scanning electron micrographs demonstrating the surface topography of the denuded basement membrane from limbal zone (Fig. 2). This technique of EDTA removal of epithelium allows visualisation not only of these extrusions of stroma, but also of denuded folds of stroma forming the palisades of Vogt (Fig. 2A). In rabbits the interdigitation of stroma and epithelium is much less pronounced, and 'pegs' or papillae were not seen by scanning elecron microscopy of denuded rabbit limbus (Fig. 3). Only occasional indentations or pits into the stroma were noted (Fig. 3B).

Hemidesmosomes along the cell membrane of the basal cells and their processes appear similar in structure to those of other stratified squamous epithelia (Fig. 1), although the percentage of basal cell membrane occupied by hemidesmosomes is significantly less than that found in central cornea in both humans and rabbits (Table I). Two predominant features of the matrix in the 'pegs' are basement membrane and an accumulation of anchoring fibrils (Fig. 1). Basement membrane within the 'pegs' is duplicated even in young human eyes (Fig. 1). In addition, small discontinuities are pres- ent in the basement membrane. The area of basement membrane $/ 100 \mu \mathrm{m}$ of basal cell membrane is not significantly different to that found in central cornea in humans but was significantly different in rabbit (Table). In humans, there were differences, however, in the pattern of duplication of the basement membrane. Not only were there regions of duplication of basement membrane within the 'pegs' between cytoplasmic processes, but in the limbus segments of basement membrane and associated anchoring fibrils were found some distance away from the base of the cytoplasmic processes (Fig. 4). Between these layers of basement membrane, collagen fibrils, small vesicles and anchoring fibrils intervened. Within the matrix 'pegs', individual anchoring fibrils extend from the basement membrane and intersect other fibrils (Fig. 1A), which in turn either loop back to the basement membrane a distance away or extend deeper into the stroma beyond the level of the processes. Anchoring plaques are present in stromal matrix near the base of the cytoplasmic extensions (Fig. 1B). The cross-banded anchoring fibrils can be seen coursing into the plaques.

Immunolocalisation of laminin in the limbal basement membrane zone showed the same irregular interdigitation seen by electron and light microscopy in both humans and rabbits (Fig. 5). In some sections, the antibody could be localised to short segments between the basement membrane and blood vessels (Fig. 5a). The pattern of localisation of type VII collagen was similar to that of laminin, except that the band of localisation beneath the epithelium appeared to be wider. As with laminin, small segments of type VII collagen were present between the basement membrane and blood vessels (Fig. 5d). These segments of laminin and type VII collagen may correspond to the areas of duplication of these components found deeper in the human stroma (Fig. 4). Small fluorescent dots or beadlike structures of type VII collagen were observed in both human and rabbit limbus. These structures may correspond to adhesion plaques.

\section{Discussion}

Although the components of the adhesion complex of the corneal and limbal epithelium are the same, their patterns of arrangement 

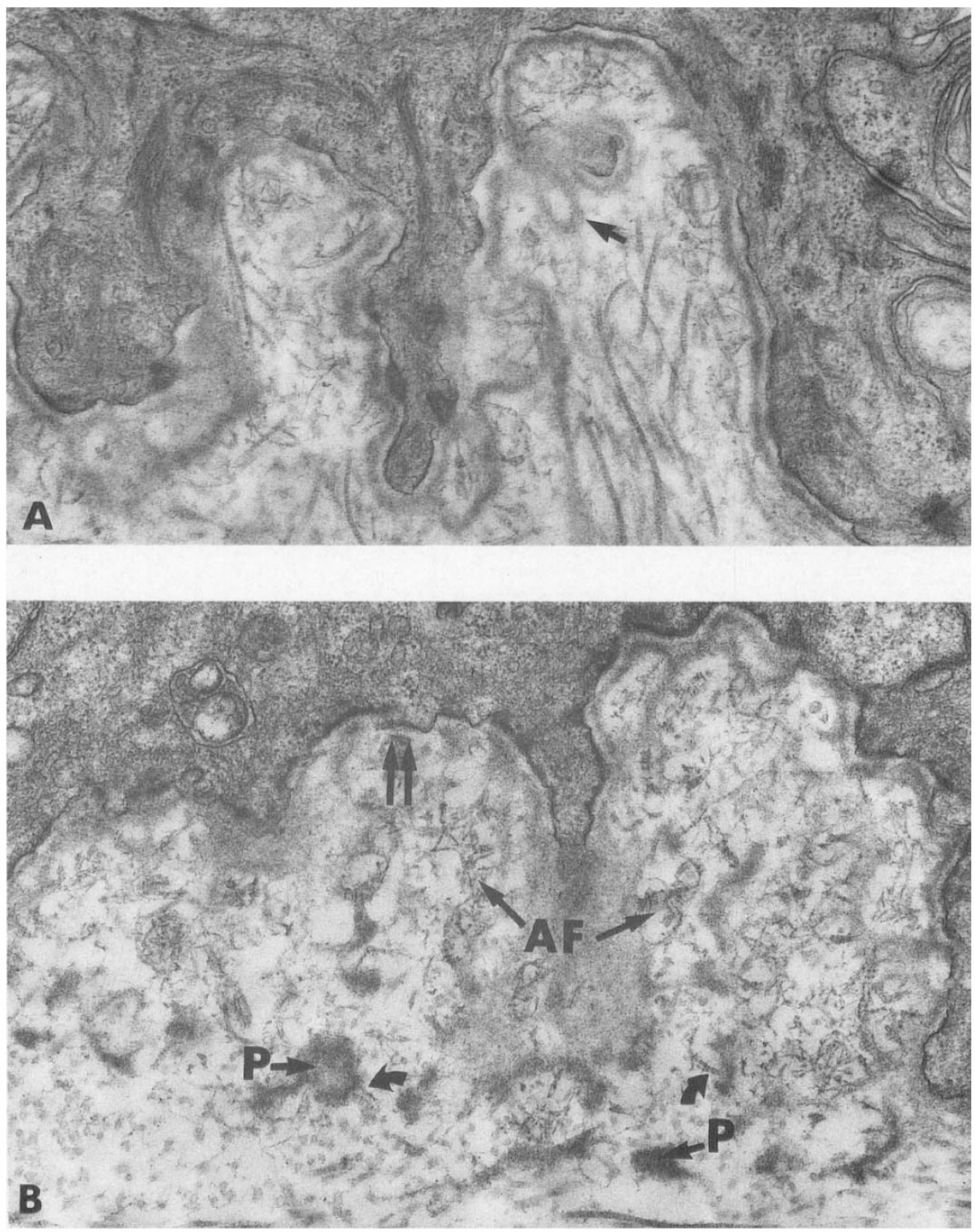

Fig. 1. Electron micrographs of basement membrane zone of human limbal epithelium from (A) a 19-year-old, and $(B)$ a 57-year-old. In both, the interdigitation of cytoplasmic extensions with 'pegs' of stroma are obvious. Duplication or looping of basement membrane within the 'pegs' (arrow) (A) and short discontinuties in the basementmembrane (double arrow) (B) are present. Anchoring fibrils extend from the basement membrane and intersect with other anchoring fibrils, some of which loop back to the basement membrane. A complex network of anchoring fibrils ( $A F$ 's) is a major component of the stromal 'pegs'. In B, cross-banded anchoring fibrils (curved arrows) insert into anchoring plaques $(P)$. ( $A$ and $B, X 34,000)$. 

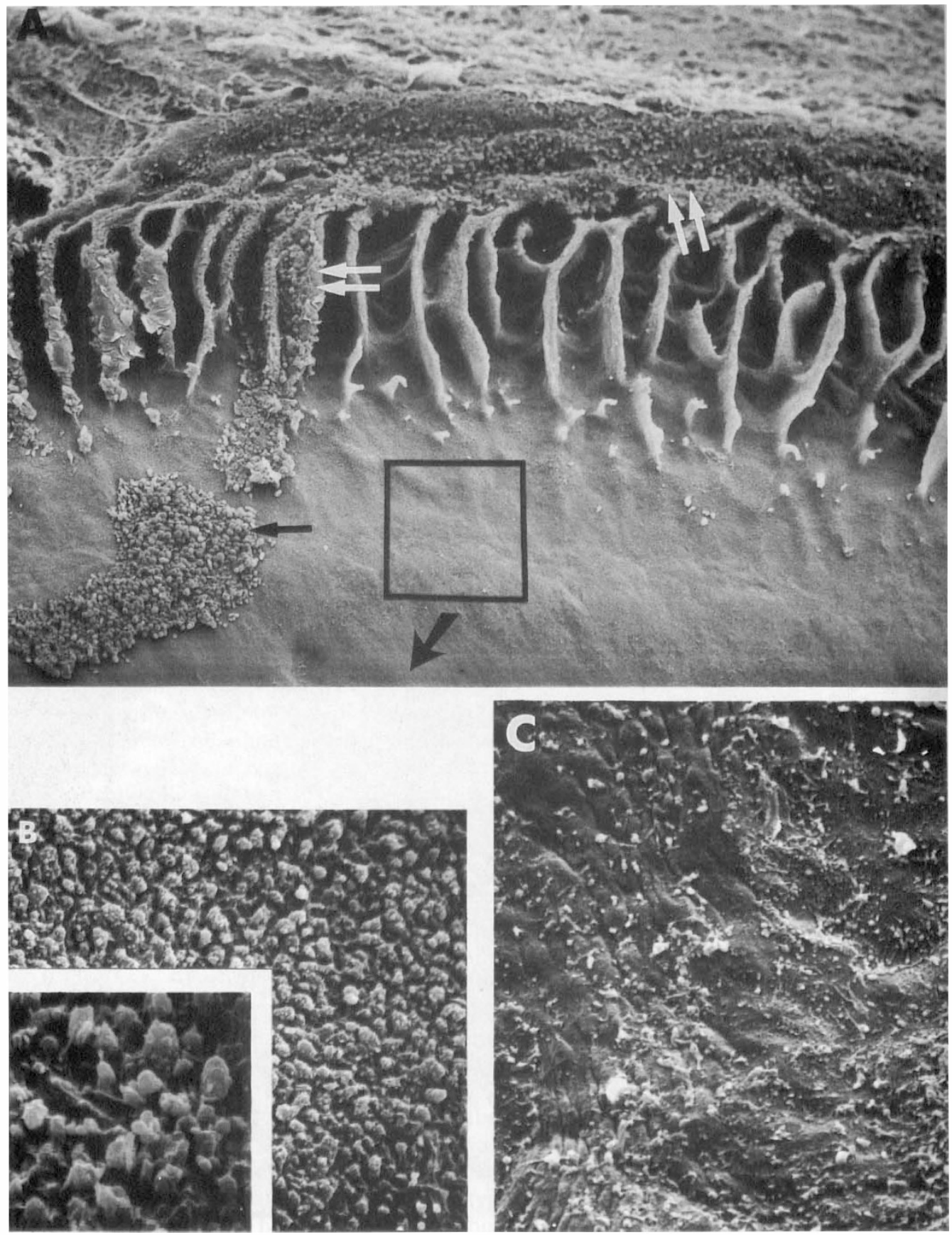

Fig. 2. A. Low-power scanning electron micrograph of limbal region from a 57-year-old human; the epithelium had been partially removed by incubation in the presence of EDTA. Folds of the Palisades of Vogt are obvious. Patches of remnant epithelium are present at the lower left (arrow), on the surfaces of the Palisades of Vogt at the left (arrows), and over the tops of the Palisades distal from the cornea (arrows) (X77). B. Higher-power micrograph from region of denuded limbus indicated by square in A. Note 'pegs' or papillae protruding from the stroma. Compare $B$ to denuded basement membranes from central cornea in $C$. ( $B$ and $C, X 2000)$ Note relative flatness of cornea basement membrane compared to limbus. Inset in B shows 'pegs' at higher magnification (X5100). 

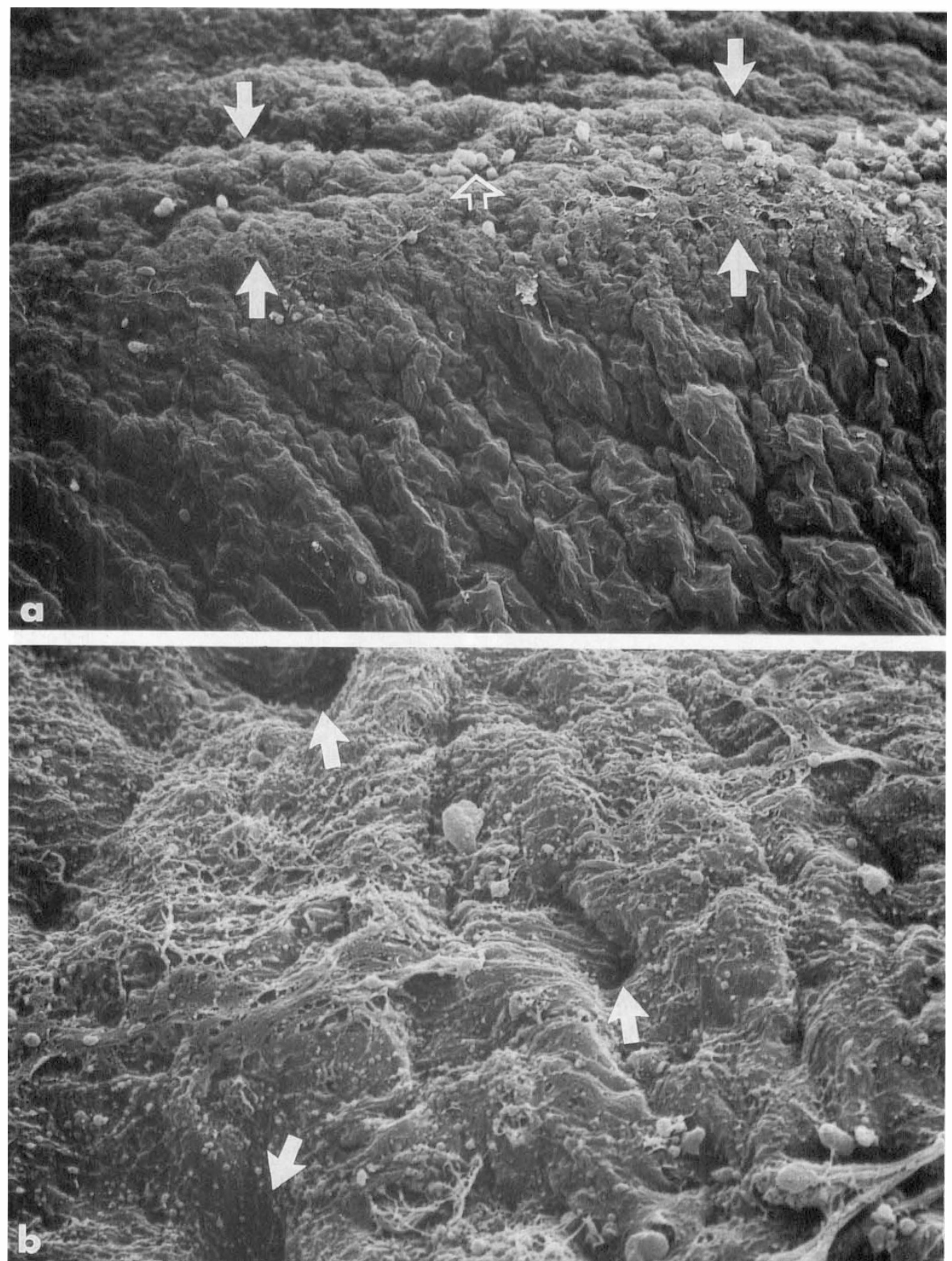

Fig. 3. Scanning electron micrographs of rabbit limbal region denuded of epithelium. a. Low-power magni fication of limbal zone which runs through the micrograph (between arrows). Note lack of Palisades of Vogt. This area has a few remnant cells (open arrow). Folds in region are artifacts of critical point drying (X260). b. Higher magnification of limbal region. Occasional pits into the stroma are present (arrows), but 'pegs' or papillae noted in humans are lacking $(X 2,600)$. 
Table Percentage of basal cell membrane occupied by $H D$ and area of BM/100 um basal cell membrane in limbus and central cornea

\begin{tabular}{ccc} 
Per cent of & Area $(\mathrm{mm})$ of \\
basal cell & BM/100 um \\
membrane & basal cell \\
$N$ & occupied by HD & membrane \\
\hline
\end{tabular}

\begin{tabular}{lrrr} 
Limbus & & & \\
Human & 10 & $14.9 \pm 3.5^{\mathrm{a}}$ & $25.2 \pm 7.5^{\mathrm{c}}$ \\
Rabbit & 2 & $16.1 \pm 2.6^{\mathrm{b}}$ & $17.0 \pm 3.0^{\mathrm{d}}$ \\
Central cornea & & & \\
Human & 16 & $27.9 \pm 9.2^{\mathrm{a}}$ & $29.0 \pm 21.0^{\mathrm{c}}$ \\
Rabbit & 7 & $33.4 \pm 4.8^{\mathrm{b}}$ & $12.0 \pm 0.1^{\mathrm{d}}$ \\
\hline
\end{tabular}

$\mathrm{N}=$ number, $\mathrm{HD}=$ hemidesmosomes, $\mathrm{BM}=$ basement membrane.

a,b,d $\mathrm{P}<0.01$; $^{\mathrm{c}}$ not significant. differ in several ways. First, the percentage of basal cell membrane occupied by hemidesmosomes is less in the limbus than in the central cornea in both rabbit and human. One generally correlates amount of adhesion with amount of adhesion junctions. Either there is less abrasion to the epithelium in the limbus, requiring fewer junctions, or the interdigitation of the basal cell cytoplasmic processes with the stromal matrix compensates for the smaller number of hemidesmosomes.

A second difference is the pattern of duplication of the basement membrane, seen particularly in corneas of older humans. In the central cornea, duplication is usually a dense accumulation of basement membrane-like material with pockets of anchoring fibrils

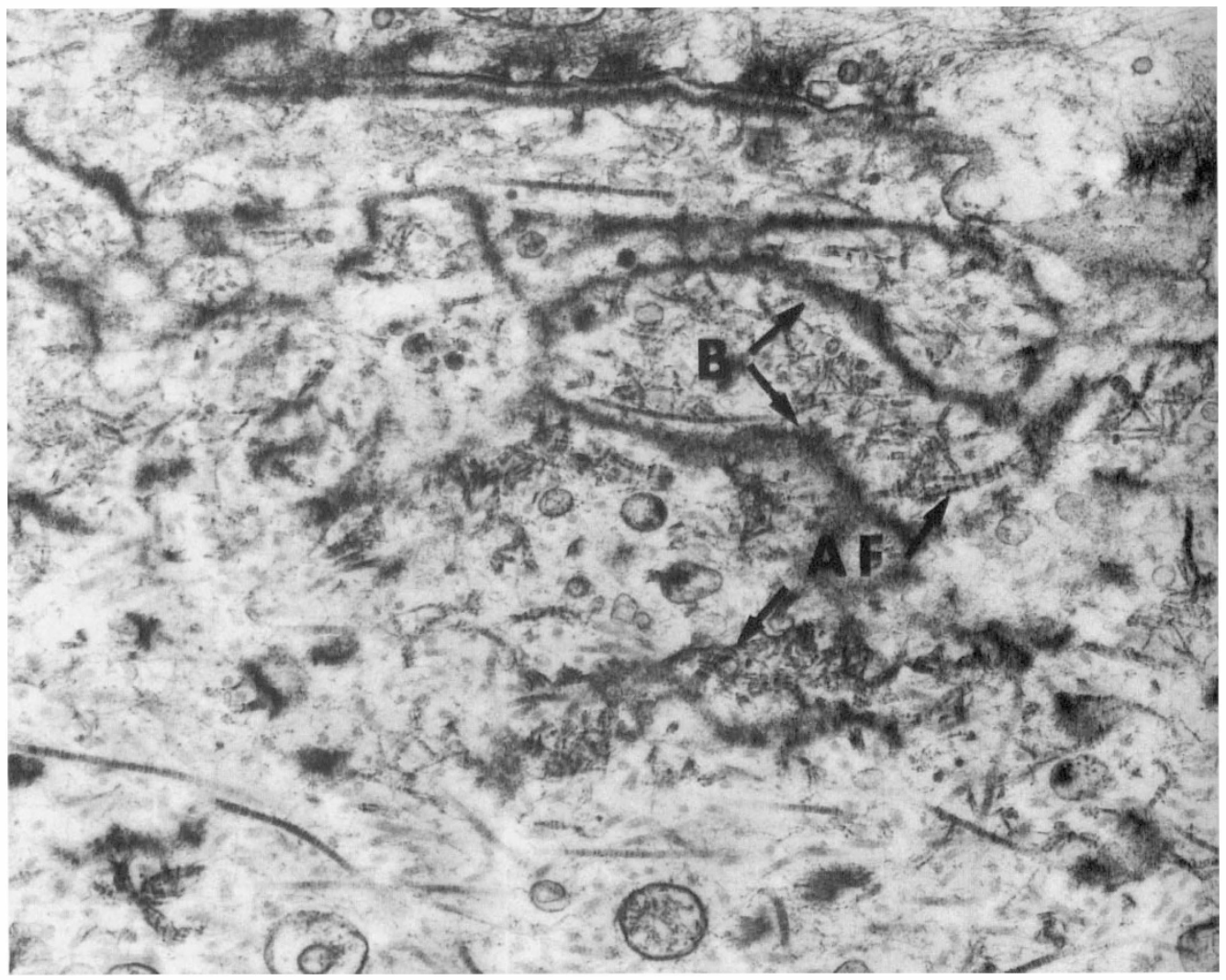

Fig. 4. Electron micrograph from limbal region of a 77-year-old human demonstrating duplicated basement membrane $(B)$ with associated anchoring fibrils $(A F)$ located more deeply in the stroma. Small vesicles, collagen fibrils, and anchoring fibrils fill the space between separated layers of duplicated basement membrane. These duplicated basement membrane zone components may be comparable to the short segments of immunolocalised laminin and type VII collagen seen in Figs. 5a and $d(X 31,000)$. 

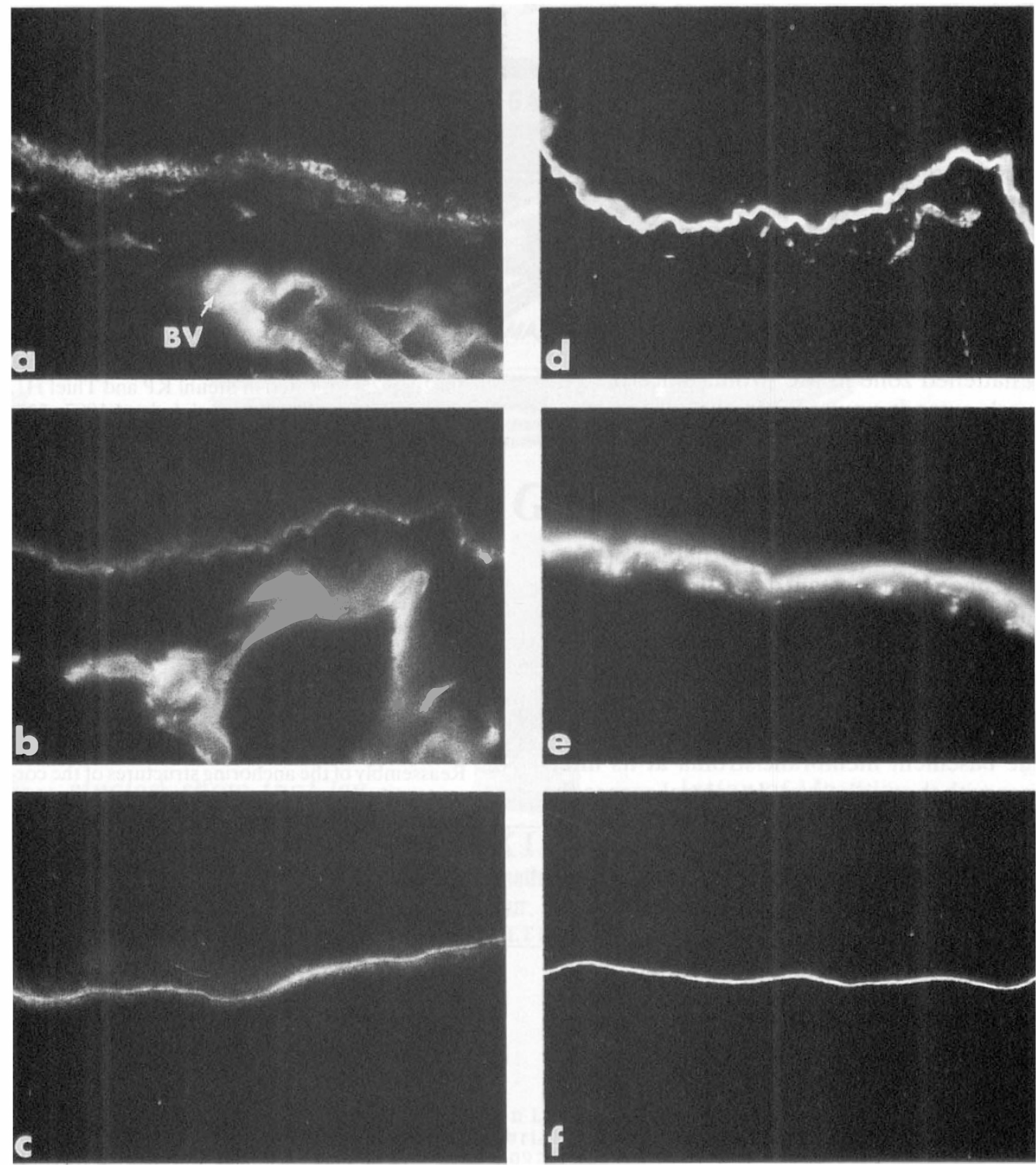

Fig. 5. Light micrographs demonstrating immunolocalisation of laminin (a-c) and type VII collagen (d-f) in human limbus $(a, d)$, rabbit limbus $(b, e)$, and human central cornea $(c, f)$. In both human and rabbit limbus, the patterns of immunolocalisation of laminin and type VII are an irregular convoluted fluorescent band; in central cornea, the pattern is simpler. Note in the human sections $(a, d)$ the segments of laminin and type VII collagen located deeper in the stroma between the basement membrane zone and blood vessels $(B V),(a, b, e, X 750 ; c, d, f$, X300).

embedded in the thickened basement membrane. ${ }^{14}$ In the limbus, the layers of duplication are interspersed with larger amounts of stromal material including type I collagen fibrils. Such duplication does not seem to be enhanced by ageing to the degree that it is in central cor- nea. This may mean that basal cells of the limbus synthesise these extracellular matrix (ECM) components sporadically, in addition to the basement membrane and anchoring fibrils, leaving isolated islands of basement membrane in the stroma. 
Another difference between limbus and central cornea in both species is the arrangement of the anchoring fibril network. In the cornea, anchoring fibrils insert into the flattened basement membrane and splay out into the anterior stroma, terminating distally in anchoring plaques. Thus, the complex network of interweaving fibrils is a zone that extends parallel to the basement membrane to a depth of approximately $1-1.5 \mu \mathrm{m}$ into the stroma. Compared with the central cornea, the anchoring fibril network in the limbus is less of a flattened zone in the stroma since the network extends upward into the matrix 'pegs' which are surrounded by cytoplasmic extensions. Thus, although the anchoring fibrils terminate distally in anchoring plaques, the plaques do not appear to be as deep within the stroma and the anchoring fibril network is not a flat zone with a constant depth but has undulating borders. This characteristic is reminiscent of the undulating basement membrane found in the epidermis. ${ }^{15}$

Finally, a major difference between limbus and central cornea is the surface topography of the basement membrane/stroma at its interface with the epithelium. In central cornea the basement membrane is relatively flat in both human and rabbit, whereas the limbal surface is far more irregular with the presence of 'pegs' or papillae and the folds of the Palisades of Vogt in the human. One can hypothesise that this complex surface of the human limbus provides more basal cell surface area in contact with the stromal region in which the vascular supply is present. Thus, increased nutrients required by the avascular adjacent regions or the ocular surface could be absorbed or transferred, respectively.

Humans may require such an increased basement membrane surface area because Bowman's layer may impede movement of nutrients in the anterior stroma. Since rabbits do not have a Bowman's layer, basal surfaces of central cornea may have readier access to nutrients with less movement of nutrients required of the limbal epithelium which therefore has a less complex basement membrane surface topography.

The expert technical assistance of Sandra Spurr-
Michaud, Ann Tisdale, Laila Hanninen and Ellen Zieske is gratefully acknowledged.

\section{References}

${ }^{1}$ Kinoshita S, Kiorpes TC, Friend J, Thoft RA: Limbal epithelium in ocular surface wound healing. Invest Ophthalmol Vis Sci 1982; 23: 73-80.

${ }^{2}$ Schermer A, Galvin S, Sun TT: Differentiationrelated expression of a major $64 \mathrm{~K}$ corneal keratin in vivo and in culture suggests limbal location of corneal epithelial stem cells. J Cell Biol 1986; 103: 49-62.

${ }^{3}$ Lütjen-Drecoll E, Steuhl KP, Arnold WH: Morphologische Besonderheiten der Conjunctiva bulbi. In: Marquardt R (ed), Chronische Conjunctivitis-trockenes Auge. Springer, Vienna, 1982, pp 25-34. Cited in Steuhl KP and Thiel HJ, Graefe's Arch Clin Exp Ophthalmol 1987; 225: 53-8.

${ }^{4}$ Bukusoglu G and Zieske JD: Characterisation of a monoclonal antibody that specifically binds basal cells in the limbal epithelium. Invest Ophthalmol Vis Sci 1988, 29: (ARVO Suppl): 192.

${ }^{5}$ Gipson IK, Spurr-Michaud SJ, Tisdale AS: Anchoring fibrils form a complex network in human and rabbit cornea. Invest Ophthalmol Vis Sci 1987; 28: 212-20.

${ }^{6}$ Gipson IK, Spurr-Michaud SJ, Tisdale AS: Hemidesmosomes and anchoring fibril collagen appear synchronously during development and wound healing. Dev Biol, 1988; 126: 253-62.

${ }^{7}$ Gipson IK, Spurr-Michaud S, Tisdale A, Keough M: Reassembly of the anchoring structures of the corneal epithelium during wound repair in the rabbit. Invest Ophthalmol Vis Sci, 1989; 30: 69-78.

${ }^{8}$ Keene DR, Sakai LY, Lunstrum GP, Morris NP, Burgeson RE: Type VII collagen forms an extended network of anchoring fibrils. J Cell Biol 1987; 104: 611-21.

${ }^{9}$ Fujikawa LS, Foster CS, Gipson IK, Colvin RB: Basement membrane components in healing rabbit corneal epithelial wounds: immunofluorescence and ultrastructural studies. J Cell Biol 1984; 98: $128-38$.

${ }^{10}$ Gipson IK, Grill SM, Spurr SJ, Brennan SJ: Hemidesmosome formtion in vitro. J Cell Biol 1983; 97: 849-57.

${ }^{11}$ Burgeson RE, Morris NP, Murray LW, Duncan KG, Keene DR, Sakai LY: The structure of type VII collagen. Ann NY Acad Sci 1985; 460: 47-57.

${ }^{12}$ Lunstrum GP, Sakai LY, Keene DR, Morris NP, Burgeson RE: Large complex globular domains of type VII procollagen contribute to the structure of anchoring fibrils. J Biol Chem 1986; 261: 9042-8.

${ }^{13}$ Hogan MJ, Alvarado JA, Weddell JE: Histology of the human eye. Philadelphia, W. B. Saunders, 1971: 128-9.

${ }^{14}$ Alvarado J, Murphy C, Juster R: Age-related changes in the basement membrane of the human corneal epithelium. Invest Ophthalmol Vis Sci 1983; 24: 1015-28.

${ }^{15}$ Kawabe TT, MacCallum DK, Lillie JH: Variation in basement membrane topography in human thick skin. Anat Rec 1985; 211: 142-8. 